\title{
LA IGUALDAD DE OPORTUNIDADES EN LA NUEVA CONSTITUCIÓN EUROPEA
}

\author{
EQUAL OPPORTUNITIES IN THE NEW EUROPEAN CONSTITUTION
}

Cinta Castillo

Universidad de Sevilla

\section{RESUMEN:}

La igualdad entre hombres y mujeres es un Derecho fundamental que aparece en la nueva Constitución europea. En este estudio se recogen los diferentes artículos de este documento de la Unión Europea que dan prueba de la legalidad de igualdad de oportunidades entre ambos sexos.

Palabras clave:

Artículo, Unión Europea, igualdad, Carta.

\section{Abstract:}

Equality between men and women is a fundamental Right which appears in the new European Constitution. In this study we recollect the different articles from this document of the European Union which demonstrate the legality of equal opportunities between both sexes.

\section{KeYwORDS:}

Article, European Union, equality, Charter. 
La igualdad y no discriminación entre hombres y mujeres es un Derecho fundamental, reconocido en el Art. 14 de nuestra Carta Magna, que ha tenido hasta ahora un nivel aceptable de protección en el derecho vigente de la Unión Europea.

Se ha materializado en normas que regulan determinadas manifestaciones de la igualdad, así entre las de derecho vinculante podemos destacar las relativas a la igualdad de remuneraciones por un trabajo de igual valor, la igualdad en el empleo, la conciliación entre la vida profesional y familiar, la prohibición del acoso sexual en el ámbito laboral, la perspectiva de género en los fondos estructurales.

Como normas de carácter orientador, encontramos las que se dirigen a favorecer a representación equilibrada de hombres y mujeres en la toma de decisiones, o la no adopción de normas relativas a la violencia de género.

El Proyecto de Tratado de una Constitución para Europa se presenta con lagunas en cuanto a la eficacia y garantía de derechos fundamentales, como lo es la igualdad entre hombres y mujeres.

La Carta de los Derechos Fundamentales de la Unión europea puede dar lugar a una pérdida del status jurídico de la igualdad como derecho, para pasar a darle la categoría de principio.

La técnica legislativa empleada por la Convención para el futuro de Europa en relación con los derechos fundamentales se resume, en la reproducción de las cláusulas de estos derechos que ya existían en los Tratados comunitarios, añadiendo los derechos de la Carta de Derechos Fundamentales, pero con la adición de nuevos criterios interpretativos.

Se establece como cláusula de interpretación que "aquellos derechos fundamentales resultantes de las tradiciones constitucionales comunes de los Estados miembros que se reconozcan en la presente Carta se interpretarán en armonía con las citadas tradiciones".

Esto significa que va a tener un nivel de protección menor que la ofrecida por el Derecho Comunitario o por los Tratados internacionales.

No está claro sí esta cláusula de interpretación es o no aplicable a la igualdad entre hombres y mujeres, puesto que en ella se introduce la división entre derechos y principios, pero sin determinar cuáles son unos y otros. Por ello, podríamos interpretar que no afecta a la igualdad y que este derecho mantiene su carácter fundamental. Sin embargo existen razones que nos llevan a la confusión, como que la regulación de la conciliación familiar y profesional incluya elementos de un derecho y de un principio, sin especificar cuáles son unos y otros, pero afirmando que "los derechos 
deben respetarse, mientras que los principios deben observarse". (Referencia a Teresa Freixes).

El pasado 6 de abril, se formuló una Declaración en el curso de la reunión extraordinaria de la Conferencia de las Comisiones Parlamentarias sobre la igualdad de oportunidades de hombres y mujeres en la U. E.

Esta conferencia, organizada por la Comisión de derechos de la mujer e igualdad de oportunidades del Parlamento Europeo, abordó asuntos que inciden de lleno en el futuro, destacamos tres bloques que resumen las quince propuestas de la Declaración:

* En cuanto al Proyecto de Tratado Constitucional, se incide en la necesidad de garantizar la igualdad de oportunidades en la Constitución.

* En cuanto a la representación equilibrada de hombres y mujeres, se advierte de la persistente infrarepresentación de las mujeres en los procesos de decisión, que constituye una deficiencia fundamental para la sociedad europea.

* En cuanto a la legislación en materia de igualdad de oportunidades fuera del mercado laboral, deja patente que determinados Estados miembros continúan sin decidirse a adoptar las medidas necesarias para combatir cualquier discriminación por razón de sexo fuera del ámbito del empleo.

En este sentido, recordar que nuestra Ley integral contra la violencia hacia las mujeres es la primera que se aprueba en Europa, por tanto la legislación para condenar y prevenir la violencia como máximo exponente de la desigualdad entre hombres y mujeres, sigue siendo en Europa la gran asignatura pendiente.

A pesar de que los datos disponibles no permiten, en rigor, la comparación entre los distintos Estados miembros en cuanto a la situación real de la violencia que sufren las mujeres, es indudable que para la Unión Europea en su conjunto la violencia de género es un problema de graves consecuencias y raíces muy profundas.

La cuestión, efectivamente, preocupa a los gobiernos y sobre todo a la sociedad civil organizada, a los medios de comunicación...etc., pero está todavía muy lejos de ser una prioridad política en el seno de la Unión Europea.

Es muy importante, por lo tanto, que la Constitución Europea establezca una base jurídica para la elaboración de una política común.

El texto más importante que se ha aprobado recientemente, en relación con este tema en el Parlamento Europeo, es la posición Común del Consejo para aprobar un Programa de Acción Comunitario 2004-2008, para prevenir y combatir la violencia ejercida sobre los niños, jóvenes y mujeres y proveer a las víctimas y grupos de riesgo, a través del programa Daphne II. 
El avance contra la lacra social que supone la tortura y el asesinato de las mujeres, exige la igualdad entre hombres y mujeres. Sólo alcanzando la igualdad real se podrá erradicar la violencia de género, pues el origen se encuentra en la desigualdad, en la dependencia y en la discriminación que sufren las mujeres.

La Resolución del Parlamento Europeo sobre la política de la Unión Europea en materia de igualdad de género en torno al 8 de marzo, hace las siguientes manifestaciones:

* Solidaridad con todas las mujeres que sean privadas de su dignidad como seres humanos, que son víctimas de violencia, violaciones, mutilación sexual, trata de seres humanos y explotación sexual, y cualquier otra forma de discriminación.

* Reconoce que la Unión europea ha desempeñado un papel político esencial en los últimos 30 años a la hora de promover la igualdad entre mujeres y hombres. Solicitando al Consejo que asuma una iniciativa en el nivel de las Naciones Unidas para organizar la Conferencia de Bejing +10 .

* Pone de manifiesto que hay Estados que aun incorporando las Directivas sobre cuestiones de género en sus legislaciones respectivas no adoptan medidas de apoyo necesarias para lograr la igualdad real en la vida diaria. Así se destaca en el Informe anual de la Comisión sobre disparidades por razón de sexo, en el que se confirma la desigualad existente entre hombres y mujeres.

* Se hace un llamamiento a los 25 Estados para que presenten una lista de tres candidatos con representantes de ambos sexos para su designación como Comisarios, como se decidió en la convención Europea.

* Se pide a la Comisión, al Consejo y a los Estados miembros que den un lugar prioritario en su agenda política a los derechos de la mujer y la igualdad de oportunidades, y señala que los nuevos Estados han de realizar esfuerzos adicionales para invertir la tendencia actual de declive en la participación de la mujer en la vida social, política y económica.

* Se insiste en la necesidad de crear un "Instituto europeo para las cuestiones de género", ya solicitado al Parlamento.

* Se solicita al Consejo y a la Comisión que declaren el año 2006 Año europeo contra la violencia ejercida hacía las mujeres.

* Se encarga al Presidente que transmita esta Resolución al Consejo, a la Comisión, al Secretario General de Naciones Unidas, así como a los Gobiernos de los Estados miembros. 
Para fortalecer la Política de la Unión sobre Igualdad, debemos reforzar las herramientas jurídicas necesarias para desarrollar una política global, mediante una integración coherente de la dimensión de la igualdad en todos los ámbitos.

Podemos entender que la igualdad entre hombres y mujeres figura entre los objetivos de la Unión. Así se expresa en el Art. 3, además de disponer de su artículo específico dentro del apartado de las políticas y el funcionamiento de la Unión, apartado III, Art. 2.

Esta presencia de la igualdad entre los objetivos, permitirá un mayor refuerzo competencial de la Unión Europea en esta materia, lo que deberá ser ampliado y mejorado si se incorpora a los valores. Sin embargo, es necesario que cuanto antes dispongamos de una Directiva sobre Violencia de Género.

Análisis del estado de garantía de derechos reconocidos en lo que se refiere a la igualdad de oportunidades entre hombres y mujeres. Igualdad de Género en el Proyecto de Constitución Europea En octubre de 2002 se presentó una Contribución del grupo socialista al Anteproyecto de Tratado Constitucional, elaborada por Josep Borrell, Carlos Carnero, Diego López Garrido y Elena Valenciano, con una serie de reivindicaciones en materia de género que se recogieron en gran medida. La futura Constitución europea representa un avance muy importante con respecto a los Tratados anteriores en cuanto a la igualdad entre hombres y mujeres. Lo más importante es que gracias a esta Constitución la igualdad entre hombre y mujer se convierte en un valor y en un objetivo de la Unión Europea.

De forma esquemática, la protección de la igualdad de género queda como sigue:

* Incorporación en la Constitución Europea de la igualdad entre hombres y mujeres como uno de los valores de la Unión. En su Artículo I-2 se especifica la igualdad entre mujeres y hombres como uno de los valores comunes de los Estados miembros. Quedando de este modo como un valor fundamental para la democracia europea.

* Se impone como objetivo a la Unión promover la igualdad entre el hombre y la mujer: "La Unión combatirá la exclusión social y la discriminación y fomentará la justicia y la protección sociales, la igualdad entre mujeres y hombres, la solidaridad entre las generaciones y la protección de los derechos del niño"

(Artículo I-3).

* La Carta de Derechos Fundamentales de la UE se incluye en la parte II Tratado de Constitución. El Tratado por el que se establece un Constitución para Europa otorgará, pues, a la Carta pleno valor constitucional a partir de su entrada en vigor, y le conferirá carácter jurídicamente vinculante. 
En su Artículo II-83, se reconoce explícitamente el derecho a la igualdad entre mujeres y hombres. Como consecuencia, la igualdad entre mujeres y hombres deberá garantizarse en todos los ámbitos de la UE (cláusula transversal), inclusive en materia de empleo, trabajo y retribución.

También se recoge que este principio deigualdad de trato no impide el mantenimiento o adopción de medidas que ofrezcan ventajas concretas destinadas a facilitar al sexo menos representado el ejercicio de una actividad profesional o a prevenir o compensar desventajas en sus carreras profesionales.

El texto también reconoce, en aras de la conciliación de la vida familiar y profesional, el derecho de toda persona a ser protegida frente a cualquier despido por causa relacionada con la maternidad (Artículo II-93).

* En su parte III, de las Políticas y el funcionamiento de la Unión, hay que destacar el Artículo III-116 en el que se pone de manifiesto que en todas las acciones contempladas en esta parte de la Constitución (es decir, las políticas de la UE) la Unión tratará de eliminar las desigualdades entre la mujer y el hombre y de promover su igualdad.

* La unión apoyará y completará la acción de los Estados miembros en la igualdad entre mujeres y hombres por lo que respecta a las oportunidades en el mercado laboral y al trato en el trabajo. Y se establece que la ley marco europea (nueva categoría, en la que participará por codecisión el $\mathrm{PE}$ ) podrá establecer normas mínimas que habrán de aplicarse progresivamente (Artículo III-210).

* Queda recogida la igualdad de retribución sin discriminación por razón de sexo, y la ley o ley marco europea establecerá medidas para garantizar este principio (Artículo III-214).

* Especial relevancia tienen los Artículo III-267 y Artículo III-271, por los que la Unión luchará contra la trata de seres humanos y la explotación sexual, en particular de mujeres y niños, garantizado por las medidas establecidas por la ley o ley marco europea.

* A destacar por su novedad e importancia, es la Declaración de la Conferencia Intergubernamental en la que se conviene que, en su empeño general por eliminar las desigualdades entre la mujer y el hombre, la Unión tratará en sus distintas políticas de combatir la violencia doméstica en todas sus formas. Indicando que es preciso que los Estados miembros adopten todas las medidas necesarias para prevenir y castigar estos actos delictivos y para prestar apoyo y protección a las víctimas (Declaración relativa al Artículo III-116).

Principales referencias sobre mujer - igualdad en la Constitución Europea: 


\section{ARTÍCULO I-2}

Valores de la Unión

La Unión se fundamenta en los valores de respeto de la dignidad humana, libertad, democracia, igualdad, Estado de Derecho y respeto de los derechos humanos, incluidos los derechos de las personas pertenecientes a minorías. Estos valores son comunes a los Estados miembros en una sociedad caracterizada por el pluralismo, la no discriminación, la tolerancia, la justicia, la solidaridad y la igualdad entre mujeres y hombres.

\section{ARTÍCULO I-3}

\section{Objetivos de la Unión}

1. La Unión tiene como finalidad promover la paz, sus valores y el bienestar de sus pueblos.

2. La Unión ofrecerá a sus ciudadanos un espacio de libertad, seguridad y justicia sin fronteras interiores y un mercado interior en el que la competencia sea libre y no esté falseada.

3. La Unión obrará en pro del desarrollo sostenible de Europa basado en un crecimiento económico equilibrado y en la estabilidad de los precios, en una economía social de mercado altamente competitiva, tendente al pleno empleo y al progreso social, y en un nivel elevado de protección y mejora de la calidad del medio ambiente. Asimismo, promoverá el progreso científico y técnico. La Unión combatirá la exclusión social y la discriminación y fomentará la justicia y la protección sociales, la igualdad entre mujeres y hombres, la solidaridad entre las generaciones y la protección de los derechos del niño.

\section{ARTÍCULO II-83}

Igualdad entre mujeres y hombres

La igualdad entre mujeres y hombres deberá garantizarse en todos los ámbitos, inclusive en materia de empleo, trabajo y retribución. El principio de igualdad no impide el mantenimiento o la adopción de medidas que supongan ventajas concretas en favor del sexo menos representado.

\section{PARTE III DE LAS POLÍTICAS Y EL FUNCIONAMIENTO DE LA UNIÓN TÍTULO I DISPOSICIONES DE APLICACIÓN GENERAL}

\section{ARTÍCULO III-115}


La Unión velará por la coherencia entre las diferentes políticas y acciones contempladas en la presente Parte, teniendo en cuenta el conjunto de sus objetivos y observando el principio de atribución de competencias.

\section{ARTÍCULO III-116}

En todas las acciones contempladas en la presente Parte, la Unión tratará de eliminar las desigualdades entre la mujer y el hombre y de promover su igualdad.

\section{ARTÍCULO III-210}

1. Para alcanzar los objetivos mencionados en el artículo III-209, la Unión apoyará y complementará la acción de los Estados miembros en los siguientes ámbitos:

a) la mejora, en particular, del entorno de trabajo, para proteger la salud y la seguridad de los trabajadores;

b) las condiciones de trabajo;

c) la seguridad social y la protección social de los trabajadores;

d) la protección de los trabajadores en caso de resolución del contrato laboral;

e) la información y la consulta a los trabajadores;

f) la representación y la defensa colectiva de los intereses de los trabajadores y de los empresarios, incluida la cogestión, sin perjuicio del apartado 6;

g) las condiciones de empleo de los nacionales de terceros países que residan legalmente en el territorio de la Unión;

h) la integración de las personas excluidas del mercado laboral, sin perjuicio del artículo III-283;

i) la igualdad entre mujeres y hombres por lo que respecta a las oportunidades en el mercado laboral y al trato en el trabajo;

j) la lucha contra la exclusión social;

k) la modernización de los sistemas de protección social, sin perjuicio de la letra c).

2. A efectos del apartado 1:

a) la ley o ley marco europea podrá establecer medidas destinadas a fomentar la cooperación entre los Estados miembros mediante iniciativas para mejorar los conocimientos, desarrollar el intercambio de información y buenas prácticas, promover planteamientos innovadores y evaluar experiencias, con exclusión de toda armonización de las disposiciones legales y reglamentarias de los Estados miembros; 
b) en los ámbitos mencionados en las letras a) a i) del apartado 1, la ley marco europea podrá establecer normas mínimas que habrán de aplicarse progresivamente, teniendo en cuenta las condiciones y reglamentaciones técnicas existentes en cada Estado miembro. Dicha ley marco europea evitará establecer trabas de carácter administrativo, financiero y jurídico que obstaculicen la creación y el desarrollo de pequeñas y medianas empresas. En todos los casos, la ley o ley marco europea se adoptará previa consulta al Comité de las Regiones y al Comité Económico y Social.

\section{ARTÍCULO III-214}

1. Cada Estado miembro garantizará la aplicación del principio de igualdad de retribución entre trabajadoras y trabajadores por el mismo trabajo o por un trabajo de igual valor.

2. A efectos del presente artículo,se entiende por "retribución" el salario o s ueldo normal de base o mínimo,y cuales quiera otras gratificaciones satisfechas,directa o indirectamente, en dinero o en especie, por el empresario al trabajador en razón de la relación laboral. La igualdad de retribución, sin discriminación por razón de sexo, significa:

a) que la retribución establecida para un mismo trabajo remunerado por unidad de obra se fije con arreglo a una misma unidad de medida;

b) que la retribución establecida para un trabajo remunerado por unidad de tiempo sea igual para un mismo puesto de trabajo.

3. La ley o ley marco europea establecerá las medidas para garantizar la aplicación del principio de igualdad de oportunidades e igualdad de trato entre mujeres y hombres en asuntos de empleo y ocupación, incluido el principio de igualdad de retribución por un mismo trabajo o por un trabajo de igual valor. La ley o ley marco se adoptará previa consulta al Comité Económico y Social.

4. Con objeto de garantizar en la práctica la plena igualdad entre mujeres y hombres en la vida profesional, el principio de igualdad de trato no impedirá a ningún Estado miembro mantener o adoptar medidas que ofrezcan ventajas concretas destinadas a facilitar al sexo menos representado el ejercicio de una actividad profesional o a prevenir o compensar desventajas en sus carreras profesionales.

\section{ARTÍCULO III-267}

1. La Unión desarrollará una política común de inmigración destinada a garantizar, en todo momento, una gestión eficaz de los flujos migratorios, un trato equitativo de los nacionales de terceros países que residan legalmente en los Estados miembros, así 
como una prevención de la inmigración ilegal y de la trata de seres humanos y una lucha reforzada contra ambas.

2. A los efectos del apartado 1, la ley o ley marco europea establecerá las medidas en los ámbitos siguientes:

a) las condiciones de entrada y residencia y las normas relativas a la expedición por los Estados miembros de visados y permisos de residencia de larga duración, incluidos los destinados a la reagrupación familiar;

b) la definición de los derechos de los nacionales de terceros países que residan legalmente en un Estado miembro, con inclusión de las condiciones que rigen la libertad de circulación y de residencia en los demás Estados miembros;

c) la inmigración y residencia ilegales, incluidas la expulsión y la repatriación de residentes en situación ilegal;

d) la lucha contra la trata de seres humanos, en particular de mujeres y niños.

3. La Unión podrá celebrar con terceros países acuerdos para la readmisión, en sus países de origen o de procedencia, de nacionales de terceros países que no cumplan o que hayan dejado de cumplir las condiciones de entrada, presencia o residencia en el territorio de uno de los Estados miembros.

4. La ley o ley marco europea podrá establecer medidas para fomentar y apoyar la acción de los Estados miembros destinada a propiciar la integración de los nacionales de terceros países que residan legalmente en su territorio, con exclusión de toda armonización de las disposiciones legales y reglamentarias de los Estados miembros.

5. El presente artículo no afectará al derecho de los Estados miembros a establecer volúmenes de admisión en su territorio de nacionales de terceros países procedentes de terceros países con el fin de buscar trabajo por cuenta ajena o por cuenta propia.

\section{ARTÍCULO III-271}

1. La ley marco europea podrá establecer normas mínimas relativas a la definición de las infracciones penales y de las sanciones en ámbitos delictivos que sean de especial gravedad y tengan una dimensión transfronteriza derivada del carácter o de las repercusiones de dichas infracciones o de una necesidad particular de combatirlas según criterios comunes.

Estos ámbitos delictivos son los siguientes: el terrorismo, la trata de seres humanos y la explotación sexual de mujeres y niños, el tráfico ilícito de drogas, el tráfico ilícito de armas, el blanqueo de capitales, la corrupción, la falsificación de medios de pago, la delincuencia informática y la delincuencia organizada. 
Teniendo en cuenta la evolución de la delincuencia, el Consejo podrá adoptar una decisión europea que determine otros ámbitos delictivos que respondan a los criterios previstos en el presente apartado. Se pronunciará por unanimidad, previa aprobación del Parlamento Europeo.

\section{ARTÍCULO $_{23} 2$}

Igualdad entre mujeres y hombres

La igualdad entre mujeres y hombres deberá garantizarse en todos los ámbitos, inclusive en materia de empleo, trabajo y retribución. El principio de igualdad no impide el mantenimiento o la adopción de medidas que supongan ventajas concretas en favor del sexo menos representado.

1 Artículo II-82 de la Constitución. 2 Artículo II-83 de la Constitución.

\section{EXPLICACIÓN}

El primer párrafo de este artículo se ha basado en el artículo 2 y en el apartado 2 del artículo 3 del Tratado CE, que han sido sustituidos por los artículos I-3 y III-116 de la Constitución que imponen como objetivo a la Unión promover la igualdad entre el hombre y la mujer en la Unión, y en el apartado 1 del artículo 141 del Tratado CE, que ha sido sustituido por el apartado 1 del artículo III-214 de la Constitución. Se inspira en el artículo 20 de la Carta Social Europea revisada, de 3 de mayo de 1996, y en el punto 16 de la Carta Comunitaria de los Derechos Sociales Fundamentales de los Trabajadores.

Se basa asimismo en el apartado 3 del artículo 141 del Tratado CE, sustituido por el apartado 3 del artículo III-214 de la Constitución, y en el apartado 4 del artículo 2 de la Directiva 76/207/CEE del Consejo relativa a la aplicación del principio de igualdad de trato entre hombres y mujeres en lo que se refiere al acceso al empleo, a la formación y a la promoción profesionales, y a las condiciones de trabajo.

El segundo párrafo recoge en una fórmula más breve el apartado 4 del artículo III214 de la Constitución conforme al cual el principio de igualdad de trato no impide el mantenimiento o la adopción de medidas que ofrezcan ventajas concretas destinadas a facilitar al sexo menos representado el ejercicio de una actividad profesional o a prevenir o compensar desventajas en sus carreras profesionales. De conformidad con el apartado 2 del artículo 52 1, el segundo párrafo no modifica el apartado 4 del artículo III-214.

\section{DECLARACIÓN RELATIVA AL ARTíCULO III-116}


La Conferencia conviene en que, en su empeño general por eliminar las desigualdades entre la mujer y el hombre, la Unión tratará en sus distintas políticas de combatir la violencia doméstica en todas sus formas. Es preciso que los Estados miembros adopten todas las medidas necesarias para prevenir y castigar estos actos delictivos y para prestar apoyo y protección a las víctimas.

\section{ARTÍCULO $_{33} 1$}

Vida familiar y vida profesional

1. Se garantiza la protección de la familia en los planos jurídico, económico y social.

2. Con el fin de poder conciliar vida familiar y vida profesional, toda persona tiene derecho a ser protegida contra cualquier despido por una causa relacionada con la maternidad, así como el derecho a un permiso pagado por maternidad y a un permiso parental con motivo del nacimiento o de la adopción de un niño.

1 ARTíCULO II-93 de la Constitución

\section{ExPLICACIÓN}

El apartado 1 del artículo 331 se basa en el artículo 16 de la Carta Social Europea. El apartado 2 se inspira en la Directiva 92/85/CEE del Consejo relativa a la aplicación de medidas para promover la mejora de la seguridad y de la salud en el trabajo de la trabajadora embarazada, que haya dado a luz o en período de lactancia, y en la Directiva 96/34/CE relativa al Acuerdo marco sobre el permiso parental celebrado por la UNICE, el CEEP y la CES. Se basa asimismo en el artículo 8 (protección de la maternidad) de la Carta Social Europea y se inspira en el artículo 27 (derecho de los trabajadores con responsabilidades familiares a la igualdad de oportunidades y de trato) de la Carta Social revisada. El término "maternidad" abarca el período desde la concepción a la lactancia.

$25 / 10 / 2004$

SEMINARIO AUDEM 15 DE NOVIEMBRE DE 2004

\section{BIBLIOGRAFÍA}

Borrell, J., Carnero, C., \& López Garrido, D. (2003). Construyendo la Constitución Europea. Crónica Política de la Convención, Madrid, Real Instituto Elcano de Estudios Internacionales y Estatégicos.

De Enterría, E. G. (1995). El proyecto de Constitución europea. Revista Española de Derecho Constitucional, (45), 9-29. 
De Lucas Martín, F. J. (2004). Perplejidades ante la" Constitución europea". Jueces para la democracia, (50), 5-10.

Gorelli Hernández, J. (2004). El proyecto de constitución europea y la protección familar. Jornadas Universitarias Andaluzas de Derecho del Trabajo y Relaciones Laborales (22ㄹ. 2004. Málaga), 491-525.

Menéndez, A. J. (2004). Esperando a la Constitución Europea. Revista Española de Derecho Constitucional, (72), 87-120. 